\title{
Global plasma-parameter simulation of Comet 67P/Churyumov-Gerasimenko approaching the Sun *
}

\author{
N. Gortsas ${ }^{1}$, U. Motschmann ${ }^{1,2}$, E. Kührt ${ }^{1}$, K.-H. Glassmeier ${ }^{3,4}$, K. C. Hansen ${ }^{5}$, J. Müller ${ }^{2}$, and A. Schmidt ${ }^{6}$ \\ ${ }^{1}$ Institute for Planetary Research, German Aerospace Center (DLR), Rutherfordstr. 2, 12489 Berlin, Germany \\ e-mail: nikolaos.gortsas@dlr.de $e^{\star \star}$ \\ 2 Institute for Theoretical Physics, TU Braunschweig, Mendelssohnstrasse 3, 38106 Braunschweig, Germany \\ Institute for Geophysics und Extraterrestrial Physics, Mendelssohnstrasse 3, 38106 Braunschweig, Germany \\ 4 Max-Planck-Institute for Solar System Research, Max-Planck-Str. 2, 37191 Katlenburg-Lindau, Germany \\ 5 Department of Atmospheric, Oceanic and Space Sciences, The University of Michigan, Ann Arbor, Michigan 48109-2143, USA \\ ${ }^{6}$ European Space Astronomy Centre, PO Box 78, 28691 Villanueva de la Caada (Madrid), Spain
}

Received 9 April 2010 / Accepted 27 July 2010

\begin{abstract}
We simulate the evolution of the plasma environment of comet 67P/Churyumov-Gerasimenko (CG), which is the target comet of the European Space Agency's (ESA) Rosetta mission, as the comet approaches the Sun. The plasma environment is calculated in three dimensions with a hybrid plasma model. The model treats the dynamics of the solar wind protons and the cometary ions in the framework of the macroparticle approach while the electrons are treated as a massless, charge-neutralizing fluid. The simulation starts at 4.2 AU and finishes at 1.3 AU. The outgassing strength of the comet is calculated from a thermal nucleus model. The model accounts for heat conduction, heat advection, gas diffusion, sublimation, and condensation processes in a porous ice-dust matrix with moving boundaries. The movement of the boundaries (Stefan problem) is accounted for by a temperature remapping technique. The maxima of the cometary ion flux and of the magnetic field in the simulation domain are presented as functions of heliocentric distance. The bow shock (BS), the ion composition boundary (ICB), and the magnetic pileup boundary (MPB) position along the Sun-comet line as a function of heliocentric distance are also discussed. A comparison of the BS position with an analytical formula yields good agreement. The MPB and the ICB along the Sun-comet line coincide.
\end{abstract}

Key words. comets: general - comets: individual: 67P/Churyumov-Gerasimenko - plasmas - magnetic fields - interplanetary medium - methods: numerical

\section{Introduction}

We simulate the plasma environment of comet 67P/ChuryumovGerasimenko (CG), which is the target of the European Space Agency's (ESA) Rosetta mission (Glassmeier et al. 2007), during its journey into the inner Solar System by combining a hybrid plasma simulation model with a thermophysical nucleus model. This combined effort allows us a unique insight into the evolution of the plasma environment of a weak comet with increasing activity (Coates \& Jones 2009).

A key input parameter of the hybrid plasma model is the outgassing strength of the comet. In the present investigation the major cometary ion species is considered, i.e. water group ions. The outgassing strength is calculated from a sophisticated nucleus model that treats different processes such as heat conduction, heat advection, gas diffusion, sublimation, and condensation with moving boundaries in a chemically diverse nucleus. For a review of the efforts in the field of thermophysical modeling of comet nuclei we refer the reader to

\footnotetext{
* A movie showing the plasma environment of CG during its approach to the Sun is available in electronic form at http: //www . aanda. org.

$\star \star$ This e-mail address is being protected from spam bots, you need JavaScript enabled to view it.
}

Prialnik et al. (2004) and the reference therein. Previous articles concerning the plasma environment of comets were performed at some fixed heliocentric distances and the outgassing strength was set to an estimated number based on observations, or else it was derived from a simple outgassing model (Lipatov et al. 2002; Motschmann \& Kührt 2006; Delamere 2006; Gortsas et al. 2009). One of the first studies of the dynamic and very complex phenomenon of a comet approaching the Sun and thereby gaining strength in its outgassing of volatile materials has been presented by Bagdonat \& Motschmann (2002). The authors studied the plasma environment of comet 46P/Wirtanen for several heliocentric distances between 3.25 AU to $1 \mathrm{AU}$ in $2 \mathrm{D}$ and 3D. Based on characteristic plasma structures, different interaction regimes were identified. An improved investigation was presented by Hansen et al. (2007), who applied the hybrid plasma model of Bagdonat \& Motschmann (2002) and the MHD model of Gombosi et al. (1996) to investigate the plasma environment of CG at four fixed heliocentric distances. This investigation has shown that important insight in to the plasma environment of comets is obtained through a kinetic approach as presented in the hybrid plasma model. Especially far from perihelion a kinetic description reveals important aspects of the solar-wind/comet interaction. 


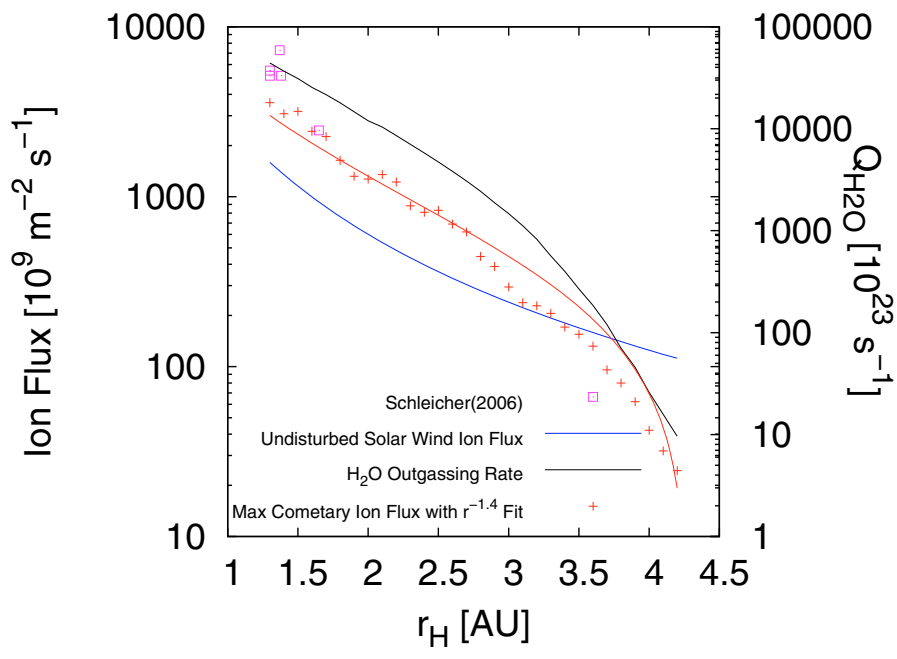

Fig. 1. Left scale: maximum of the cometary ion flux as a function of heliocentric distance. The maximum is taken from the whole simulation domain and fitted with a rational law $r^{-1.4}$. For comparison the undisturbed solar wind ion flux is also shown. Three regimes can be distinguished. At the beginning, the solar-wind ion flux exceeds the cometary ion flux, this is the test-particle regime that covers the range from 4.2 $\mathrm{AU}$ to $3.7 \mathrm{AU}$. Around 3.7 AU, these quantities reach the same values. This regime is referred to as the Mach cone regime, which covers the range up to $2.7 \mathrm{AU}$. From $2.7 \mathrm{AU}$ to $1.3 \mathrm{AU}$, the maximum cometary ion flux clealry exceeds the solar wind ion flux. This is the shock formation regime. Right scale: total water outgassing rates.

The novel feature of this study is the sophisticated thermal model employed to calculate the water activity of CG and the quasi continuous simulation of the cometary approach to perihelion. The simulation starts at 4.2 AU and finishes at 1.3 AU with a step size in heliocentric direction of $0.1 \mathrm{AU}$. In the presentation of the simulation results strong emphasis is laid on the evolution of the cometary ion flux and the magnetic field compared to the undisturbed solar wind and the interplanetary magnetic field. The simulation results are compared with analytical formulas for the BS standoff distance. Scaling laws of important boundaries are also presented.

\section{Simulation model and input parameters}

We apply a three-dimensional, quasi-neutral hybrid plasma model to study the evolution of the plasma environment of $\mathrm{CG}$ as the comet approaches the Sun. The dynamics of the solar wind protons and the cometary ions are described in the framework of the macroparticle approach while the electrons are treated as a massless, charge-neutralizing fluid. The key features of the hybrid model are discussed in preceding publications (Bagdonat \& Motschmann 2002; Gortsas et al. 2009). Therefore, only a brief overview of the main aspects for this study shall be given.

We start the calculation at 4.2 AU from scratch. After the simulation has reached quasi-stationarity, the result is taken as the starting point of the simulation at $4.1 \mathrm{AU}$. This is continued until perihelion. At each heliocentric distance the solar wind passes the simulation box up to five times. The dependence of the solar wind environment parameters, e.g. solar wind background density $n_{0}$ and the strength of the interplanetary magnetic field $B_{\text {imf }}$, are obtained through a fit of Voyager 2 and IMP 8 data (Richardson et al. 1995) and with the Parker model (Parker 1958). The corresponding curves are shown in Fig. 1 for the solar-wind ion flux and in Fig. 2 for the magnetic field strength. The increasing outgassing strength of the comet makes it necessary to adapt the size of the simulation box. This is done in a trial-and-error fashion with the aim to keep the characteristic plasma structures like bow shock approximately in the middle of the simulation box and to get a smooth transition between adjacent steps. The simulation domain increases from $1200 \mathrm{~km}$ at $4.2 \mathrm{AU}$ to $24000 \mathrm{~km}$ at perihelion.

The outgassing pattern in the hybrid code is spherically symmetric and the outgassing strength is derived from a further development of the thermophysical nucleus model of Kührt (1999). The model consists of water ice, CO ice, and dust in a porous ice-dust matrix. It solves the coupled heat and mass transfer problem with moving boundary conditions. The model takes into account erosion due to surface sublimation, which leads to the loss of internal energy stored in the eroded subsurface layers. This is achieved by applying the so-called temperature remapping technique. Following the work of Crank \& Gupta (1972) the spatial discretization is kept constant while the grid is moved according to the amount of surface sublimation. The thickness of the eroded surface layers $\Delta s$ is calculated from the Stefan condition (Stefan 1891)

$\frac{\mathrm{d} s}{\mathrm{~d} t}=\frac{Q}{\Delta H \rho}$.

$Q$ denotes the amount of surface sublimation of water ice, $\Delta H$ is the difference in thermal enthalpy, and $\rho$ the mass density of water ice. In extension to that, a non-constant number of intervals is introduced in order to be able to calculate oscillating boundaries, which can come very close to the surface. This adaptive approach is essential to model cometary activity of a multicomponent nucleus. More details of the nucleus model will be published in an upcoming article.

\section{Simulation results}

\subsection{Global view of the solar wind-comet interaction}

Part of this manuscript is a movie showing the absolute value of cometary ion flux and of the magnetic field in the orthogonal plane of $B_{\text {imf }}$ as the comet approaches the Sun. The movie is available as an online supplement.

The simulation results for the water-outgassing strength are displayed in Fig. 1. The water sublimation curve is fitted to observations of Schleicher (2006) by integrating over the whole nucleus and by assuming that $3 \%$ of the total surface is active. At 4.2 AU the comet is very faint, reaching an integrated water activity of $10^{24} \mathrm{~s}^{-1}$. At these large heliocentric distances water activity is very low. But other more volatile species can sublime from the nucleus despite low energy input from the Sun. Such a volatile species would be CO. For the present investigation, however, only water vapor is considered in the hybrid plasma model. Depending on the simulation parameters of the thermal model water exceeds CO-outgassing between 4 AU to $3.5 \mathrm{AU}$. Therefore, the role of $\mathrm{CO}$ is not further investigated here. At perihelion, the comet reaches a water-outgassing strength of $4.4 \times 10^{27} \mathrm{~s}^{-1}$. Thus, during the simulation cometary water activity increases by more then three orders of magnitude.

The weak outgassing strength at 4.2 AU yields a maximum value of the cometary ion flux, which is one order of magnitude below the undisturbed solar wind ion flux as displayed in Fig. 1. At around 3.7 AU, cometary activity has increased and yields a maximum ion flux that is comparable to the undisturbed 
N. Gortsas et al.: Global plasma-parameter simulation of Comet 67P/Churyumov-Gerasimenko approaching the Sun

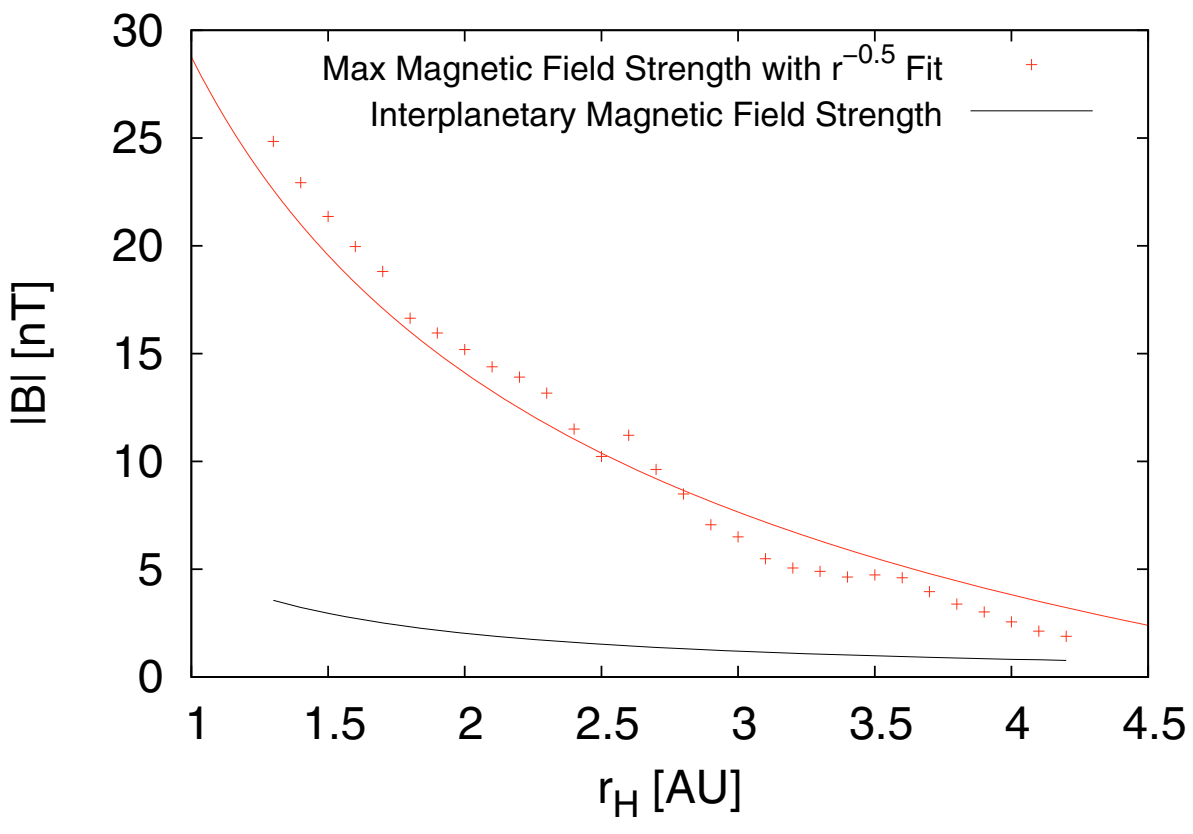

Fig. 2. Maximum of the magnetic field as function of heliocentric distance is shown. The maximum is taken from the whole simulation domain and fitted with a rational law of $r^{-0.5}$. For comparison the interplanetary magnetic field is also depicted. As the comet approaches the Sun the magnetic field maximum exceeds the $B_{\text {imf }}$ by a factor 2.5 at $4.2 \mathrm{AU}$ to a factor 7 at $1.3 \mathrm{AU}$. The enhancement of the magnetic field at $4.2 \mathrm{AU}$ is close to the nucleus surface. As the comet approaches the Sun, the maximum of the magnetic field is detached from the nucleus surface and affects large regions around the nucleus. See also Fig. 3 for the position of the magnetic pile-up region along the Sun-comet line.

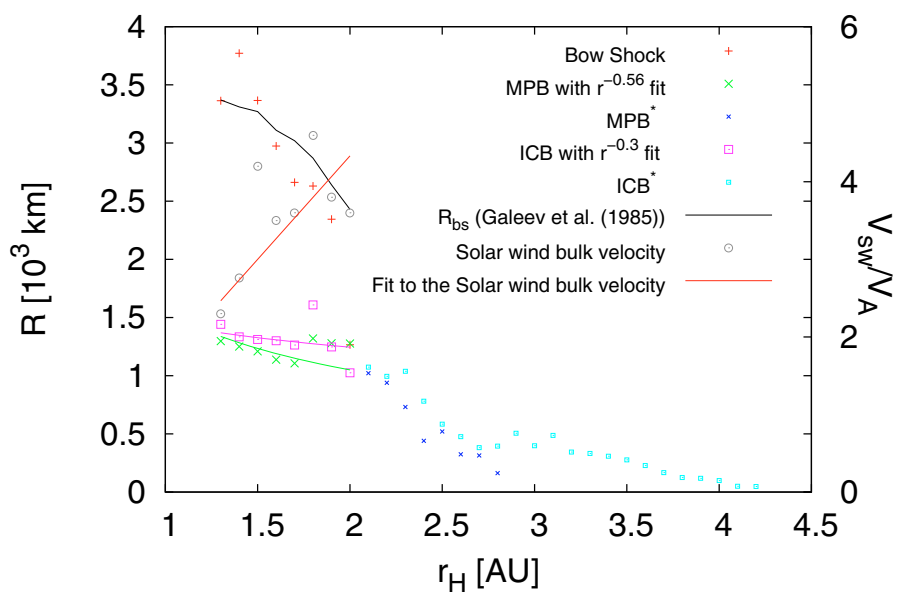

Fig. 3. Left scale: the distance of the bow shock (BS), the magnetic pileup boundary (MPB), and the ion composition boundary (ICB) to the nucleus along the Sun-comet line is displayed. The BS position agrees well with an analytical formula of Galeev et al. (1985). The MPB and the ICB are well fitted by $r_{\mathrm{H}}^{-0.56}$ and $r_{\mathrm{H}}^{-0.3}$ law, respectively. Both boundaries remain in close vicinity over the relevant heliocentric range. Beyond $2 \mathrm{AU}$ all three boundaries are not well defined because the nucleus is too weak. Therefore, the $\mathrm{MPB}^{*}$ and the $\mathrm{ICB}^{*}$ carry a superscript. They are only displayed for illustration purposes. Right scale: the absolute value of the solar-wind bulk velocity $V_{\mathrm{sw}}$ at the bow shock in units of the Alfven velocity $V_{\mathrm{A}}$, the hybrid model predicts a BS with a Mach number of about 2.3 for comet CG. As the comet approaches its perihelion position, the Mach number of the solar wind flow drops very steeply.

solar-wind ion flux. The evolution of the magnetic field is depicted in Fig. 2. At the beginning, the maximum of the magnetic field is a factor of 2.5 above the $B_{\text {imf }}$. This value is reached in front of the nucleus, while farther away the magnetic field remains undisturbed. At 3.7 AU this factor rises to a value of almost 5. The interval starting at 4.2 AU to 3.7 AU exhibits features which are characteristic to the test-particle regime as described in Bagdonat \& Motschmann (2002). The comet is too faint to cause any significant feedback to the solar wind.
The simulation data allow us to identify a second regime that covers a heliocentric range from 3.7 AU to 2.7 AU. In this interval, the growth of cometary activity is about 2 to 3 orders of magnitude. This growth process is followed by the cometary ion flux, which exceeds the solar wind flux by a factor of 2 at the end of the interval. The magnetic field exceeds the $B_{\text {imf }}$ by a factor of 7. Compared to the factor at 3.7 AU this amounts to a moderate enhancement of about 1.4. This regime has been characterized in previous studies of the solar-wind/comet interaction by the formation of the linear Mach cone (Lipatov et al. 2002; Bagdonat \& Motschmann 2002).

The last regime spans the distance from 2.7 AU to 1.3 AU. In this interval, cometary activity increases by more than one order of magnitude. The maximum of the cometary ion flux and of the magnetic field, however, seem to grow only moderately. Both quantities exceed the background values of the solar wind by a factor of 2 and 7, respectively; factors that were already reached at 2.7 AU. Hence, despite the growth in cometary activity by one order of magnitude, the maximum of the magnetic field and the cometary ion flux seem to follow the growth of the interplanetary magnetic field strength and the solar wind ion flux. In previous investigations of the solar-wind/comet interaction this last regime was characterized by the splitting of the Mach cone and the formation of a bow shock (Bagdonat \& Motschmann 2002).

The proposed division of the simulation range in three interaction regimes appears to agree well with the investigation of Hansen et al. (2007). There are also differences though. The testparticle regime is farther away from the Sun in the ranges from 4.2 AU to 3.7 AU, while in Hansen et al. (2007) this regime was at $3.25 \mathrm{AU}$. This is mainly because of different wateroutgassing curves. The formation of the Mach cone was identified by Hansen et al. (2007) at 2.7 AU, which coincides well with the present investigation. According to the present simulation the Mach cone evolves between 3.4 AU to 2.7 AU. In the global view of the solar wind-comet interaction the splitting of the Mach cone and the formation of the bow shock cannot be separated. These structures evolve from 2.7 AU to perihelion. As the comet approaches its perihelion position, the Hansen et al. (2007) and the present investigation converge as the differences in the employed water activity get smaller. 


\subsection{Shocks and cometary characteristics}

The position of the bow shock $R_{\mathrm{bs}}$ relative to the comet as function of heliocentric distance is compared with an analytical formula derived by Biermann et al. (1967). Assuming a stationary flow in an axial-symmetric $1 \mathrm{D}$ model of the solar wind the mass flux equation reads (Biermann et al. 1967)

$\frac{\mathrm{d}\left(\rho_{m} u\right)}{\mathrm{d} x}=\frac{Q m_{\mathrm{i}}}{4 \pi u_{\mathrm{g}} v^{-1} r^{2}}$.

Integrating this equation from the undisturbed solar wind conditions to the location of the bow shock leads to the following expression for the standoff distance $R_{\mathrm{bs}}$ (Biermann et al. 1967; Galeev et al. 1985)

$R_{\mathrm{bs}}=\frac{Q m_{\mathrm{i}}}{4 \pi u_{\mathrm{g}} v^{-1} \rho_{\infty} u_{\infty}\left[(\hat{\rho} \hat{u})_{\mathrm{c}}-1\right]}$.

$m_{\mathrm{i}}, u_{\mathrm{g}}, v, \rho_{\infty}$, and $u_{\infty}$ denote the cometary ion mass of the water species, the escape velocity of the ions, e.g. $1 \mathrm{~km} \mathrm{~s}^{-1}$, the ionization rate, e.g. $10^{-6} \mathrm{~s}^{-1}$, the background solar-wind proton-mass density, and velocity. $(\hat{\rho} \hat{u})_{\mathrm{c}}$ denotes the critical mass flux ratio of the contaminated solar wind for shock formation. A continuous solar wind flow is possible only until the point at which the mean molecular weight of the solar wind particles reaches a critical value. This value was estimated by Biermann et al. (1967) to be $(\hat{\rho} \hat{u})_{\mathrm{c}}=4 / 3$ based on a simplified one-dimensional model. Schmidt \& Wegmann (1982) showed that for 1P/Halley at $1 \mathrm{AU}$ a shock wave with Mach number 2 occurred at a distance from the nucleus where $(\hat{\rho} \hat{u})_{\mathrm{c}}=1.185$. Huddleston et al. (1992) employed Eq. (3) under the assumption that the critical number $(\hat{\rho} \hat{u})_{\mathrm{c}}$ is mainly a function of the cometary ion flux, while the solar wind ion flux is set to the undisturbed background values far from the comet. The authors obtained estimates for the shock position along the Sun-comet line in the range of $6.6 \times 10^{3} \mathrm{~km}$ for comet $26 \mathrm{P} / \mathrm{Grigg}$-Skjellerup with a $(\hat{\rho} \hat{u})_{\mathrm{c}}$ value of 1.22 . In the present investigation, the critical value $(\hat{\rho} \hat{u})_{\mathrm{c}}$ is used as a fit parameter. It turns out that the hybrid simulation data of the standoff distance are well fitted by Eq. (3) if $(\hat{\rho} \hat{u})_{\mathrm{c}}$ has a value of 2.05. At perihelion, the standoff distance of CG is $3.37 \times 10^{3} \mathrm{~km}$, which is a factor of 2 below the value of comet 26P/Grigg-Skjellerup, which has almost the same outgassing strength as CG at perihelion.

The standoff distance of the bow shock, the position of the ion composition boundary, and the magnetic pile-up boundary as function of heliocentric distance are displayed in Fig. 3 from 2 AU to perihelion because these boundaries are not well defined beyond $2 \mathrm{AU}$. The outgassing strength of the nucleus is too low beyond 2 AU. The ICB is defined as the location of the cross point along the Sun-comet line of the solar-wind proton density and the cometary ion density. The position of the MPB is represented by the peak value of the magnetic field strength. The ICB and MPB coincide well at perihelion, while farther away from the Sun these boundaries appear to follow different patterns. The ICB follows a $r^{-0.3}$ fit while the MPB a $r^{-0.56}$ fit. The latter is comparable to the fit of the maximum magnetic field in the simulation domain of $r_{\mathrm{H}}^{-0.5}$ as presented in Fig. 2. The coincidence of the ICB and the MPB at comet CG has also been observed at planet Mars by Bösswetter et al. (2004). The fit to the cometary ion flux maximum shown in Fig. 1 is $r_{\mathrm{H}}^{-1.4}$. A summary of the fit laws can be found in Table 1.

In Fig. 3 we also display the bow shock strength in terms of the Mach number as function of heliocentric distance. One can see that the hybrid model predicts a BS with a Mach number of
Table 1. Scaling laws.

\begin{tabular}{lcc}
\hline \hline \multicolumn{3}{c}{ 67P/Churyumov-Gerasimenko } \\
\hline$R_{\mathrm{bs}}$ & $3.4 \times 10^{3} \mathrm{~km}$ & \\
$R_{\mathrm{bs}}$ & $\sim Q$ & confirmed \\
$R_{\mathrm{mpb}}$ & $\sim r_{\mathrm{H}}^{-0.56}$ & \\
$R_{\text {icb }}$ & $\sim r_{\mathrm{H}}^{-0.3}$ & \\
$\left|F_{\mathrm{hi}}\right|_{\max }$ & $\sim r_{\mathrm{H}}^{-1.4}$ & \\
$|B|_{\max }$ & $\sim r_{\mathrm{H}}^{-0.5}$ & \\
\hline
\end{tabular}

Notes. $r_{\mathrm{H}}$ denotes heliocentric distance. Fits to the hybrid plasma simulation data are listed here. The linear dependence of the Galeev et al. (1985) formula on the outgassing strength $Q$ was confirmed by the present hybrid plasma simulation. $R$ denotes the position of the boundary from the nucleus along the Sun-comet line.

about 2.3 for comet CG. As the comet approaches its perihelion position, the Mach number of the solar wind flow drops very steeply to this value.

\section{Conclusion}

We investigate the evolution of the plasma environment of comet $\mathrm{CG}$ as the comet approaches the Sun. The simulations are performed by combining two different and sophisticated models. We calculate the plasma environment with the quasi-neutral, three dimensional hybrid plasma model. The outgassing strength of the comet, an important input parameter of the hybrid plasma model, is calculated from a thermophysical nucleus model. The thermal model solves the coupled heat- and mass transfer problem in a porous ice-dust matrix with moving boundaries (Stefan problem).

The simulation starts at 4.2 AU and finishes at 1.3 AU with a step size in heliocentric distance of $0.1 \mathrm{AU}$. The maximum of the cometary ion flux and of the magnetic field are presented as functions of heliocentric distance. As part of this investigation a movie is published, which shows the perihelion approach of CG for the cometary ion flux and the magnetic field.

The simulation results allow us to distinguish three different regimes. The test-particle regime covers a heliocentric distance between 4.2 AU to 3.7 AU. Weak mass-loading of the solar wind leads to a moderate enhancement of the magnetic field, which is localized in the vicinity of the nucleus and which reaches factors in the range 2.5 to 5 compared to the $B_{\text {imf }}$. In the Mach cone regime which covers a heliocentric distance of $3.7 \mathrm{AU}$ to about 2.7 AU, the cometary ion flux exceeds the undisturbed solarwind ion flow and leads to a stronger feedback on the solar wind parameters. The magnetic field exhibits an enhancement by a factor 7, which is detached from the nucleus surface. The last regime covers a distance of $2.7 \mathrm{AU}$ to perihelion. Although this regime shows strong effects on the plasma environment of the comet, the absolute values of the magnetic field and the cometary ion flux appear to follow the growth process of the interplanetary magnetic field and of the solar-wind ion flux as the comet approaches the Sun.

The position of the bow shock along the Sun-comet line is compared with an analytical formula by Biermann et al. (1967) and Galeev et al. (1985), which agree well.

We also presented rational law fits to the hybrid simulation data. Fits to the maximum of the cometary ion flux, the maximum of the magnetic field magnitude, and fits along the Suncomet line to the MPB, the ICB, and the BS position as function of heliocentric distance are discussed. The hybrid model predicts 
N. Gortsas et al.: Global plasma-parameter simulation of Comet 67P/Churyumov-Gerasimenko approaching the Sun

a BS with a Mach number of about 2.3. With decreasing heliocentric distance the Mach number drops very steeply.

Acknowledgements. The authors are indebted to the ISSI comet modeling team for fruitful discussions. The work of U.M. and J.M. was supported by the Deutsche Forschungsgemeinschaft under grant number MO 539/16-1. The work by K.H.G. was financially supported by the Deutsches Zentrum für Luft- und Raumfahrt and the Bundesministerium für Wirtschaft und Technologie under grant 50 QP 0402.

\section{References}

Bagdonat, T., \& Motschmann, U. 2002, Earth Moon and Planets, 90, 305 Biermann, L., Brosowski, B., \& Schmidt, H. U. 1967, Sol. Phys., 1, 254

Bösswetter, A., Bagdonat, T., Motschmann, U., \& Sauer, K. 2004, Ann. Geophys., 22, 4363

Coates, A. J., \& Jones, G. H. 2009, Planet Space Sci., 57, 1175

Crank, J., \& Gupta, R. S. 1972, J. Inst. Math. Appl., 10, 296

Delamere, P. A. 2006, J. Geophys. Res., 111, 12217

Galeev, A. A., Cravens, T. E., \& Gombosi, T. I. 1985, ApJ, 289, 807
Glassmeier, K., Boehnhardt, H., Koschny, D., Kührt, E., \& Richter, I. 2007, Space Sci. Rev., 128, 1

Gombosi, T. I., De Zeeuw, D. L., Häberli, R. M., \& Powell, K. G. 1996, J. Geophys. Res., 101, 15233

Gortsas, N., Motschmann, U., KÃijhrt, E., et al. 2009, Ann. Geophys., 27, 1555

Hansen, K. C., Bagdonat, T., Motschmann, U., et al. 2007, Space Sci. Rev., 128, 133

Huddleston, D. E., Coates, A. J., \& Johnstone, A. D. 1992, Geophys. Res. Lett., 19,837

Kührt, E. 1999, Space Sci. Rev., 90, 75

Lipatov, A. S., Motschmann, U., \& Bagdonat, T. 2002, Planet Space. Sci., 50, 403

Motschmann, U., \& Kührt, E. 2006, Space Sci. Rev., 122, 197

Parker, E. N. 1958, ApJ, 128, 664

Prialnik, D., Benkhoff, J., \& Podolak, M. 2004, Modeling the structure and activity of comet nuclei, ed. Festou, M. C., Keller, H. U., \& Weaver, H. A., 359

Richardson, J. D., Paularena, K. I., Lazarus, A. J., \& Belcher, J. W. 1995, Geophys. Res. Lett., 22, 325

Schleicher, D. G. 2006, Icarus, 181, 442

Schmidt, H. U., \& Wegmann, R. 1982, in Comet Discoveries, Statistics, and Observational Selection, ed. L. L. Wilkening, IAU Colloq., 61, 538

Stefan, J. 1891, Ann. Phys. Chem., 42, 269 